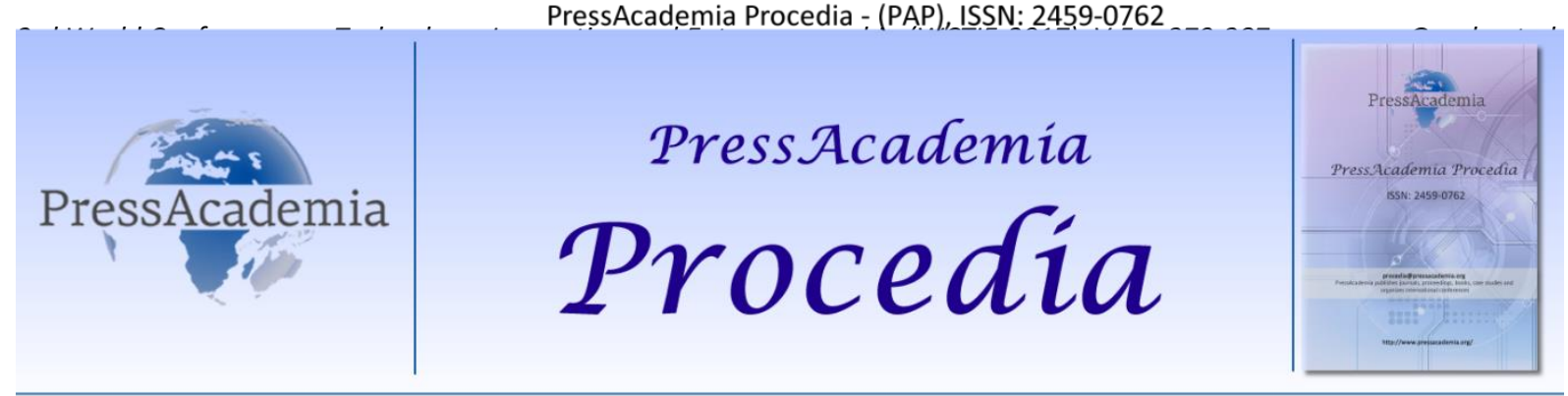

2nd World Conference on Technology, Innovation and Entrepreneurship

May 12-14, 2017, Istanbul, Turkey. Edited by Sefer Şener

\title{
SELECTION OF HANDOFF METHOD FOR SERVING AIR TRAFFIC CONTROL COMMUNICATION IN LEO SATELLITE CONSTELLATION
}

\author{
DOI: 10.17261/Pressacademia.2017.614 \\ PAP-WCTIE-V.5-2017(52)-p.379-387
}

Messaoud Garah ${ }^{1}$, Abdelali Achachi ${ }^{2}$, houcine oudira ${ }^{1}$, Djamel Benatia $^{2}$

${ }^{1}$ Department of Electrical Engineering, M'sila University, Algeria messa.gareh@gmail.com

${ }^{2}$ Department of Electrical Engineering, Batna University, Algeria

\begin{abstract}
Due to the satellite motion with respect to the earth's surface and the aircraft's mobility, it is required to handover frequently in the process of communicating, In This paper, assuming that the aircraft's location and speed can be determined, we proposes a suitable study for managing handover in satellite constellations for serving air traffic communication. The main objective of This study is selecting the best scheme which reduce significantly contact failure Probability, this techniques are: non priority, priority, and queuing handoff schemes, in this paper we are interested only in schemes that prioritize handover request in particular handoff queuing schemes. Channel allocation strategy (FCA) is employed, and the simulation results obtained concern: call blocking probability, handover failure probability, and average waiting time in the queue. Also simulation results are compared to choose the best one which reduces the contacts failureownership ratios. That supports more firm specific information is incorporated into stock prices as the ratio of foreign ownership increases.
\end{abstract}

Keywords: Air traffic control, handoff, satellite constelltion, FCA

\section{INTRODUCTION}

Due to the motion of users (aircraft in our case), more requests of handover are created, a call of a fixed or mobile user can be transferred from a satellite to another since the contact in progress cannot profit a suitable channel of communication in the current satellite, handover [1-2] establishes the transfer of connection of communication of the current channel to another.

For ensure permanent relays between ground stations and aircraft throughout the entire globe The use of satellite systems is required. To handle the increasing aircraft number, the International Civil Aviation Organization (ICAO) proposes a system of air traffic navigation reliable presented in Figure 1[3], capacitive and global based on a concept called Communication Navigation Surveillance (CNS), a system of data link: Aeronautical Telecommunication Network (ATN). The current Air Traffic Management (ATM) procedures [4] are still based on VHF communications and claims for an improvement of ATM concepts. These challenges require the development of satellite communication, navigation and surveillance systems, aiming at providing high reliability and availability system. The increasing number of disasters [5], natural or man-made, occurred during the last years, in the first hours after the disaster, the existing solutions to overcome communication problems when terrestrial infrastructures are not available are the use of satellite communication systems.

Many LEO constellations have been proposed in the literature (Iridium [6], Globalstar, etc.), while the operation of the Iridium system has given a very important experience for the study of the serious issues of these systems.

The visibility period of a satellite in LEO systems [7] can be about 5 min due to the high speed of satellites. This leads to a remarkable probability of communication interruption and the handover mechanism becomes important for the global 
performance of the system. There are two types of handover events, as is the case in land mobile systems, the cell handover [8] and the satellite handover. The first one refers to the transfer of an ongoing contact from one cell to the next one in the same satellite footprint while the second one describes the transfer of an ongoing contact from a satellite to another one.

Few studies have been carried out on the issue of satellite handover, investigating channel allocation policies for new and handover contacts using mainly fixed channel allocation (FCA) techniques. In this paper different queuing policies for handover requests were investigated in order to enhance them in air navigation satellite communication [9]. This paper is organized as follows: Section 2 presents the data model and lists the assumptions and some preliminary notions. Section 3 presents the priority strategies, Section 4 presents the simulations and discussions and the last Section will be the conclusion.

Figure 1. Simplified Block Diagram of Satellite Air Communication System

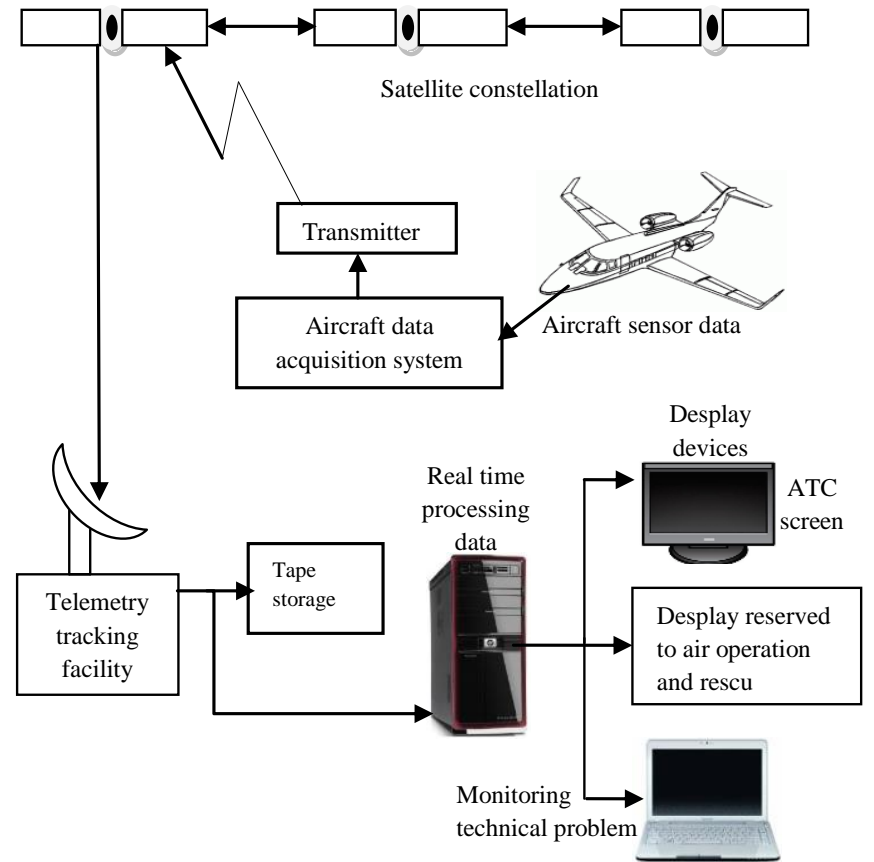

\section{DATA MODEL AND PRELIMINARY}

For the consistence with previous literatures [10] and its operational availability, the Iridium system is adopted as the basic system model. The satellite ground-track speed is approximately $26600 \mathrm{~km} / \mathrm{h}$, the Iridium satellite network is modeled as a one-dimensional environment in which mobile users move in straight lines and at constant speed.

We assume that the arrival of new contacts forms a Poisson process with an average $\lambda$ and its intensity service is $\mu$. The arrivals of handover requests, presented in Figure 2, form a Poisson process of average $\lambda \mathrm{h}$. If an aircraft is in the satellite cell, the contact duration (with mean $1 / \mu$ ) is equal to the time during which the contact is in progress.

Figure 2: Hard Handoff between the Aircraft and Satellites



a. Before handoff

b. After handoff 


\section{HANDOFF SCHEMES IN SINGLE TRAFFIC SYSTEMS}

In the coming section, we introduce no priority, priority, and queuing handoff schemes for a single traffic system such as a voice or a data system. We assume that a system has many cells, and each has $\mathrm{S}$ channels. The channel holding time have an exponential distribution with mean rate $\mu$. Both originating and handoff contacts are generated in a cell, respectively with mean rates $\lambda_{\mathrm{O}}$ and $\lambda_{H}$. We assume the system with a homogeneous cell. We concentrate our interest on a single cell (called the marked cell).

\subsection{No Priority Strategy (NPS)}

In this case, the Handover requests are handled exactly in the same way as an originating contact. So, theblocking probability of handover is equal to the probability of blocking new contacts. The NPS model is presented in Figure 3, where $\mathrm{S}$ is the number of channels present in the satellite cell .

\section{Figure 3: No Priority Strategy Scheme}

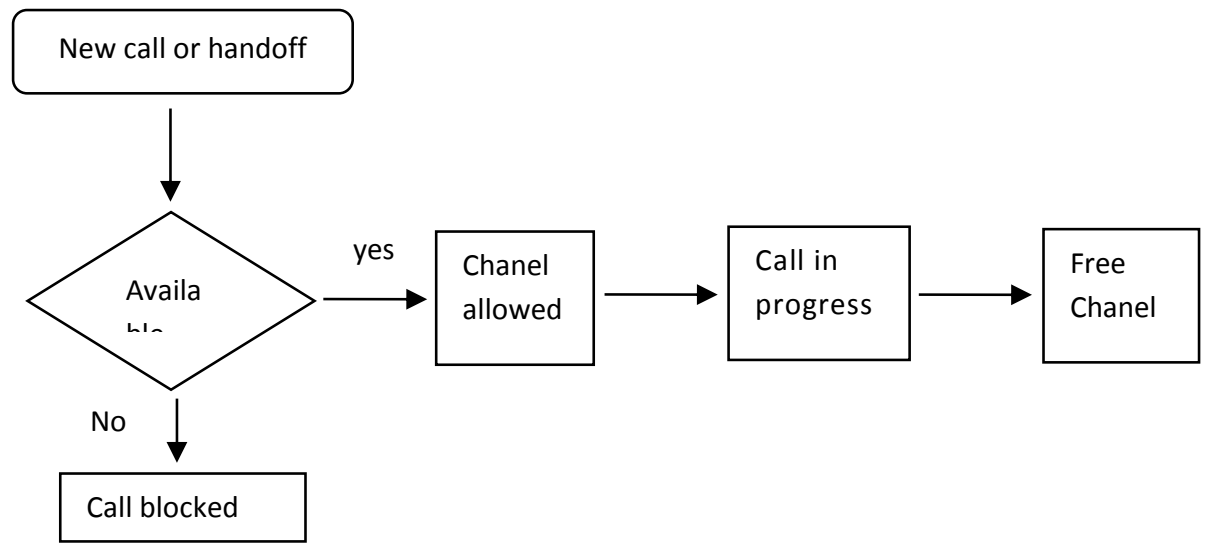

When the $S$ channels are free, they will be used by new aircraft contact or Handover. If all channels are busy the new contact will be blocked.

\section{Analytical computation}

$$
P b=P h=P s=\frac{\left(\frac{\lambda+\lambda h i}{\mu}\right)^{s}}{\sum_{k=0}^{s} \frac{\left(\frac{\lambda+\lambda h i}{\mu}\right)^{k}}{k !}}
$$

\subsection{Priority Strategy}

\subsubsection{Handoff Call Queuing Prioritizing Scheme (QPS)}

in this scheme, If all channels in the destination cell are occupied, a handover request is put in the queue as it is shown in figure 4, if a channel is released when the queue of handover request is not empty, the channel is assigned following FIFO discipline, if the received signal strength from the current BS falls below the receiver threshold level before getting service in the target cell, the call is forced to termination. 


\section{Figure 4: System Model with Priority and Queue for Handoff Contact}
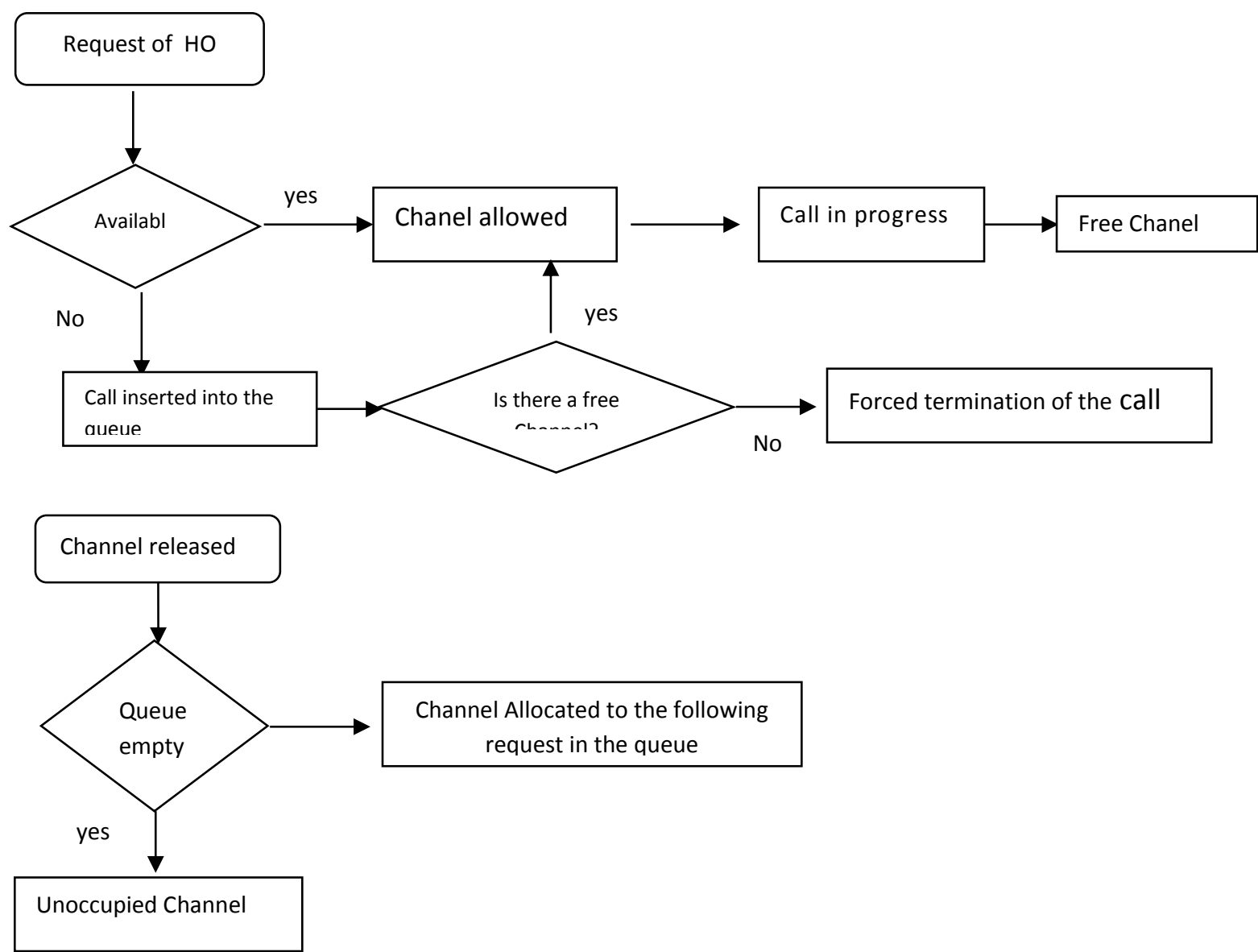

\section{Analytical computation}

$$
P_{n} \begin{cases}\frac{(\lambda+\lambda h)^{n}}{n ! \mu^{n}} P_{0} & 1 \leq n \leq s-1 \\ \frac{(\lambda+\lambda h)^{s} \lambda h^{n-s}}{s ! \mu^{s} \prod_{j=1}^{n-s}\left(s \mu+j \mu_{w}\right)} & P_{0} \quad n \geq s\end{cases}
$$

New contacts are blocked if all channels available in the satellite cell are occupied. We get :

$$
P b=\sum_{n=s}^{\infty} P_{n}
$$


In the state $\mathrm{n}$, the failure probability of handover is given by :

$$
P_{b 2 \mid n}=1-\prod_{j=0}^{n-s}\left[1-\frac{\mu_{w}}{\left(s \mu+\mu_{w}\right)^{2 j}}\right]
$$

Therefore $\mathrm{Ph}$ is given by:

$$
P h=\sum_{n=s}^{\infty} P_{b 2 \mid n} P_{n}
$$

\subsubsection{Reservation Channels Strategy (RCS) :}

Guard channels improve the probability of success of the handover by reserving a fixed number of guard channels reserved exclusively for Handover (Figure 5). The remaining channels are used for Handover and new contacts

\section{Figure 5. System Model with Priority and Reservation Channel for Handoff Contact}

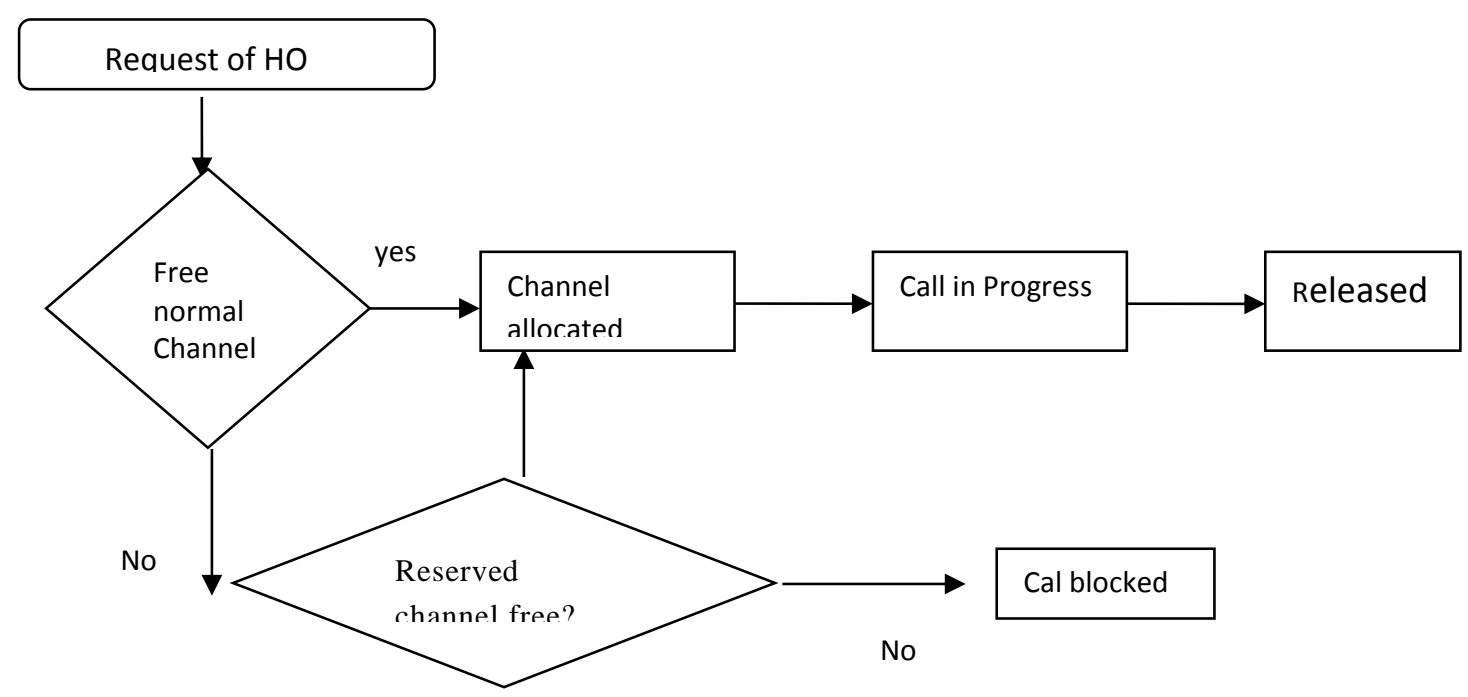

\section{Analytical computation}

$$
\begin{gathered}
P_{0}=\left[\sum_{j=0}^{n} \frac{\left(\frac{\lambda+\lambda h}{\mu}\right)^{j}}{j !}+\sum_{j=n+1}^{s} \frac{(\lambda h)^{j-n}(\lambda+\lambda h)^{n}}{j ! \mu^{j}}\right]^{-1} \\
\text { Or } a=\left(\frac{\lambda+\lambda h}{\mu}\right), \quad r=\frac{\lambda}{a \mu}
\end{gathered}
$$


The blocking probability of new contacts is equal to:

$$
P=1-\sum_{j} \frac{(a)^{j}}{j !} P_{0}
$$

The blocking probability of handover is equal to:

$$
P h=P_{0} \frac{a^{s}}{s !}(1-r)^{n}
$$

\subsubsection{Guard Channels with Queue for Handoff contact (QPS +RCS):}

It is a combination of the two previous techniques queuing requests and guard channels strategy reserved exclusively for guards Handover.

Figure 6: System Model with Reservation Channel and Queue for Handoff Contact

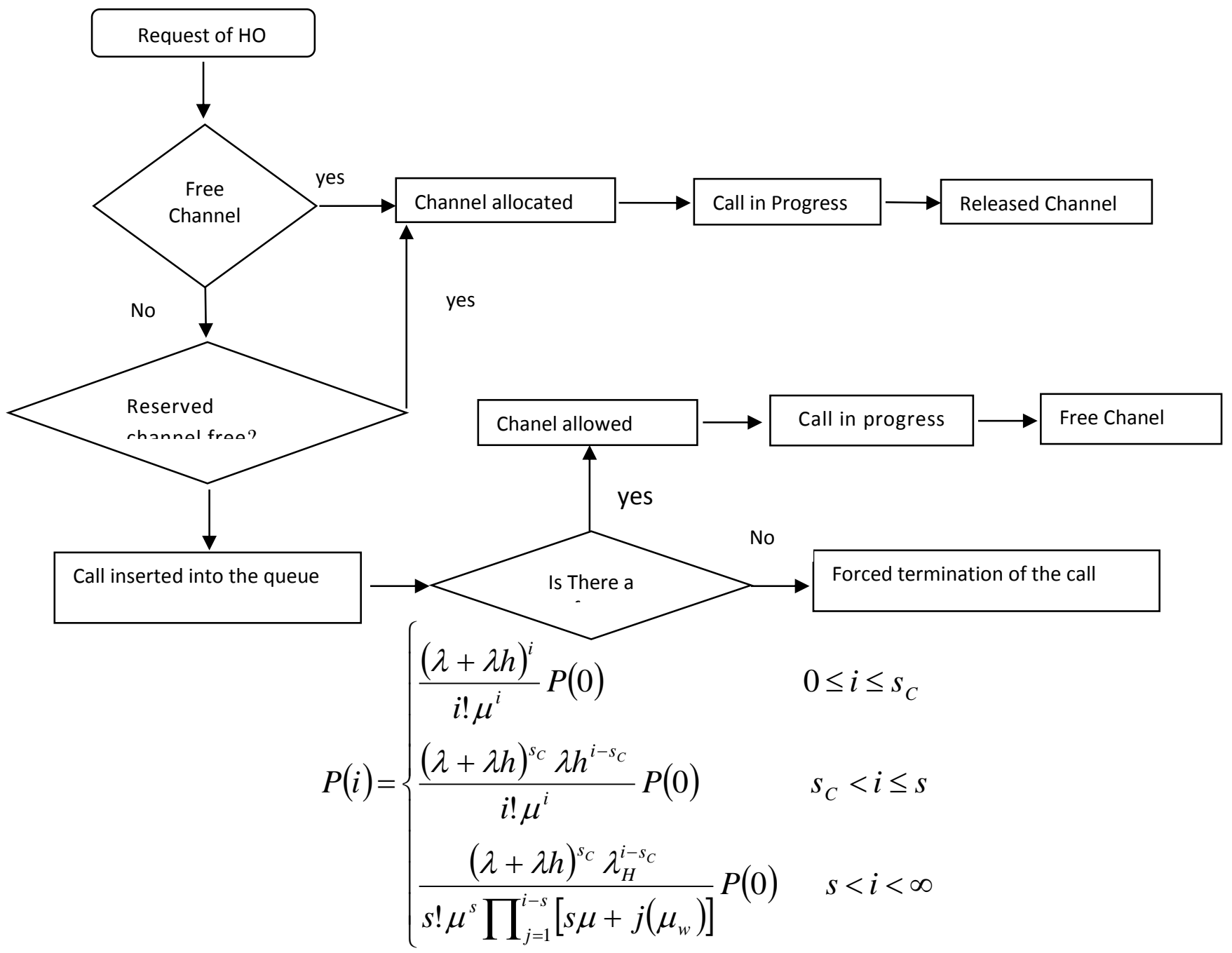


Where:

$$
P(0)=\left\{\sum_{i=0}^{S_{C}} \frac{(\lambda+\lambda h)^{i}}{i ! \mu^{i}}+\sum_{i=S_{C}+1}^{S} \frac{(\lambda+\lambda h)^{S_{C}} \lambda h^{i-S_{C}}}{i ! \mu^{i}}+\sum_{i=S+1}^{\infty} \frac{(\lambda+\lambda h)^{S_{C}}}{S ! \mu^{S}} \frac{\lambda h^{i-S_{C}}}{\prod_{j=1}^{i-S}\left[S \mu+j\left(\mu_{w}\right)\right]}\right\}^{-1}
$$

So we obtain:

$$
\begin{gathered}
P b=\sum_{i=S_{C}}^{S} P(i) \\
P h=\sum_{k=0}^{\infty} P(S+k) P_{f h \mid k} \\
P_{f h \mid k}=1-\left(\frac{\mu_{w}}{\mu S+\mu_{w}}\right) \prod_{i=1}^{k}\left\{1-\left(\frac{\mu_{w}}{\mu S+\mu_{w}}\right) \frac{1}{(2)^{i}}\right\}
\end{gathered}
$$

Where $P_{f h \mid k}$ is a probability that a handoff request fails after joining the queue in position $k+1$.

\section{Result and Discusion}

we suppose that

- $\quad$ Contact duration is exponentially distributed with a mean of $1 \mathrm{~min}$.

- The trafic in the cells follows a poisson distrubtion

- The average waiting time in the queue is exponentially distributed with a mean of 5 min.

- $\quad$ Blocked contacts are lost and cleared.

- $\quad$ The system has a total of 10 available channels per cell.

- The queue length is infinite.

- The simulation results obtained are taken after 10000 s

For the RCS model, we studied three cases according to the guard channel rates used versus to all channels allocated for each cell.
$1-\mathrm{Cr}=10 \%$
$2-\mathrm{Cr}=20 \%$
$3-\mathrm{Cr}=3$ 


\section{Figure 7: Handover Probability Failure as Function of Traffic Intensity for RCS Model}

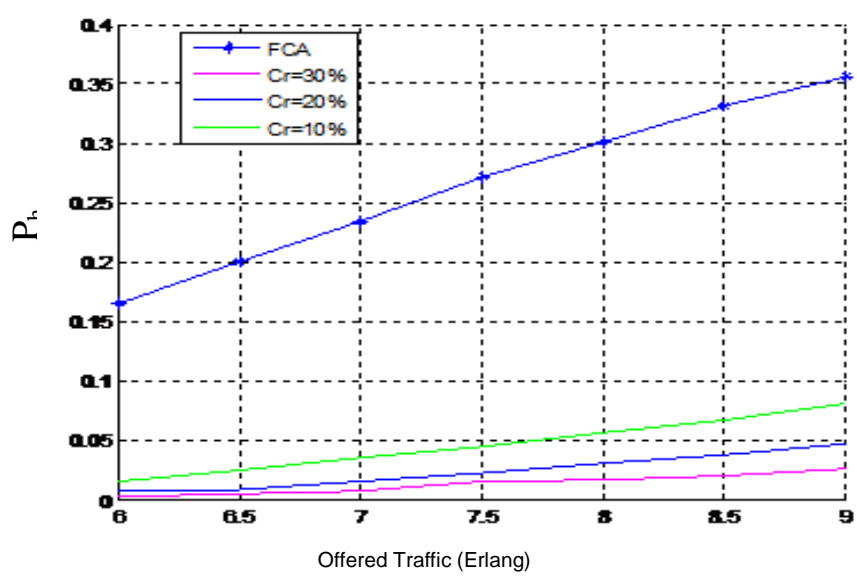

Figure 8: Handover Probability Failure as Function of Traffic Intensity for QPS +RCS t Models

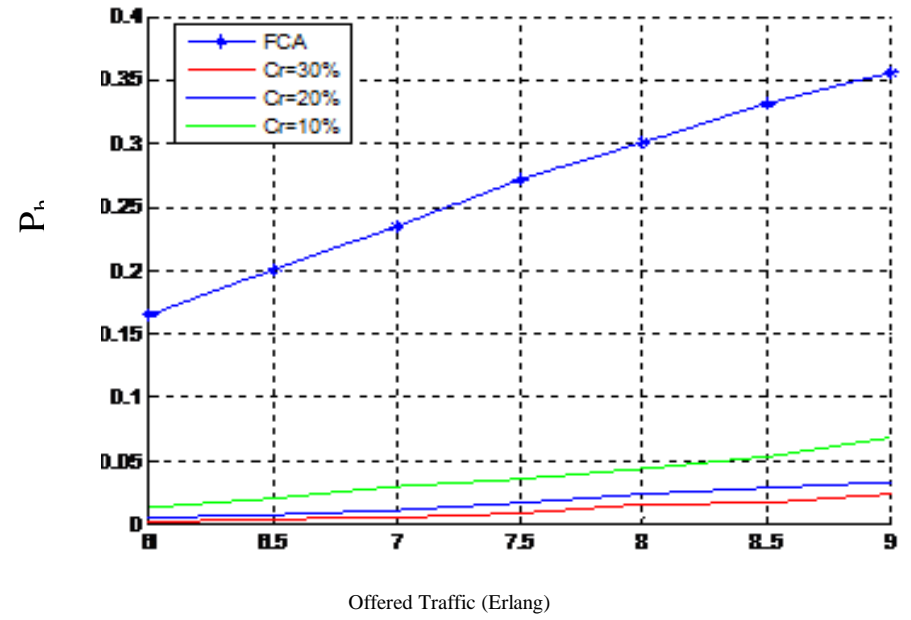

Figures (7) and (8) show handover blocking probability for the RCS and RCS + QPS models respectively depending on traffic density, in the second case (RCS+QPS )the decrease in the probability failure of handover is improved compared to the RCS model, this is due to the presence of the queue.

\section{CONCLUSION}

The handover is one of the critical procedures of communication in LEO satellite networks, the management of this mechanism must be set appropriately in order to maintain communication between pilots and controllers, and thus ensure an acceptable level of quality of communication.

In this paper we have studied a handoff schemes for satellite constellation network to reduce the probability of forced termination of ongoing contact for serving air traffic control. The scheme employs a mobile aircraft priority based on the residual time of this one in overlap region. Fixed channel allocation (FCA) strategy is adopted for simulation results. In terms of CDP (Contact dropping probability), the results show that the value of this parameter is minimized for the RCS technique, this minimization is improved when using (RCS + QPS) technique. 


\section{REFERENCES}

[1] B. Jabbari, " Teletraffic aspects of evolving and next-generation wireless communication networks," IEEE Pers. Commun. , vol. 3, Dec.1996, pp. 4-9.

[2] N. D. Tripathi, J. H. Reed and H. F. Vanlandingham, "Handoff in cellular systems" IEEE Pers. Commun., Dec. 1998, pp. $26-37$.

[3] Abdelali Achachi, Djamel Benatia, Messaoud Gareh"Satellite Handover Techniques for LEO Networks Serving Air Traffic Control Communication"International Journal of Future Generation Communication and Networking" vol 7(2014) pp 49-62

[4] G. Satapathy, J. Chen, and D. Tolani, "A traffic information service-broadcast model for mixed equipage aircraft simulationg", Integrated Communications Navigation and Surveillance (ICNS) Conference, Fairview Park, Ohio, (2010) May, pp. 1-15.

[5] M. Berioli, J. M. Chaves, N. Courville, P. Boutry, J. L. Fondere, H. Skinnemoen, H. Tork, M. Werner and M. Weinlich, “A rapidly deployable satellite backhauling system for emergency situationsg", Int. J. Satell. Commun. Network, vol. 29, (2011). pp. 419440

[6] E. D. Re, R. Fantacci, and G. Giambene, "Handover queuing strategies with dynamic and fixed channel allocation techniques in low earth orbit mobile satellite systems", IEEE Trans. Commun, vol. 47, issue 1, (1999) Jan, pp. 89-102.

[7] E. Papapetrou, S. Karapantazisny, G. Dimitriadis and F.N. Pavlidou, "Satellite handover techniques for LEO networks", Int. J. Satell. Commun. Network. vol. 22, (2004), pp. 231-245.

[8] M. Gareh and D. Benatia, "Handover prioritizing scheme for reducing call failure probability in cellular wireless network", Wireless Communications and Mobile Computing, vol. 9, (2009), pp. 1660-1667.

[9] Nour El Houda Hedjazi, Malika Ouacifi, Rachida Bouchouareb, Meriem Ourghi, Messaoud Gareh, Djamel Benatia”The Handover in the Constellations of Satellites in Low Orbit"'International Journal of Advanced Science and Technology Vol. 41, April, 2012 pp.39-48

[10] L. Chen, Q. Guo, Z. Na, K. Jiang, "A Reservation Pooling Resource Management Scheme for LEO-MSSs with Multi-class Traffic", Journal of Computational Information Systems, vol. 24, issue 8, (2012), pp. 10493-10500. 